\title{
DIFFRACTION LIMITED INFRARED IMAGES OF THE BINARY STAR T TAURI
}

\author{
A. M. Ghez, G. Neugebauer, P. W. Gorham, C. A. Haniff, S. R. Kulkarni, and K. Matthews \\ Palomar Observatory, California Institute of Technology 320-47, Pasadena, California 91125
}

C. Koresko AND S. BeCKWITH

Department of Astronomy, Cornell University, Space Sciences Building, Ithaca, New York 14850 Received 17 July 1991

\begin{abstract}
High-resolution images of $\mathrm{T}$ Tau and its infrared companion have been reconstructed from near- and mid-infrared data collected at the Hale $5 \mathrm{~m}$ telescope. The near-infrared (1-5 $\mu \mathrm{m})$ results were obtained by two dimensional speckle imaging and the mid-infrared $(10-20 \mu \mathrm{m})$ results were derived from shift and add procedures applied to slit scans. The spectral energy distributions of the separated components were constructed from 1 to $20 \mu \mathrm{m}$ data collected in less than half a year (1990 September to 1991 January). The spectral energy distribution of the optical component (T Tau N) is interpreted as containing two distinct constituents, a photosphere and a surrounding disk of circumstellar material. Measurements at a number of infrared wavelengths over the period 1985 December to 1991 January show a 2 mag color-independent change in the brightness of the infrared component ( $T$ Tau $S$ ). We propose that this may have been caused by an increase in accretion onto $\mathrm{T}$ Tau $\mathrm{S}$ and model the spectral energy distribution of $\mathrm{T}$ Tau $\mathrm{S}$ as being dominated by an accretion disk.
\end{abstract}

\section{INTRODUCTION}

In 1982, Dyck et al. (1982) discovered the first example of a close pre-main-sequence binary in the variable star $\mathrm{T}$ Tauri. Using one-dimensional speckle interferometry they measured the projected north-south binary separation to be 0 ".6. Subsequent VLA observations of T Tau at 2 and $6 \mathrm{~cm}$ showed it to be a double thermal radio source (Schwartz et al. 1984; Schwartz et al. 1986); the more intense radio component corresponded to the southern infrared source, $T$ Tau $\mathrm{S}$, while the northern source, $\mathrm{T}$ Tau $\mathrm{N}$, was identified with the visible star on the basis of astrometric studies (Hanson et al. 1983). Apart from statistical arguments (Dyck et al.), evidence for the two stars being physically related comes from the $2 \mathrm{~cm}$ VLA radio map (Schwartz et al. 1986), in which an emission bridge apparently connects the two sources. Nisenson et al. (1985) reported an additional optical component, T Tau O', 0"3 north of T Tau N, but Gorham et al. (1991) do not detect this component in their 1990 data.

To understand the T Tau system and its spectral energy distribution it is necessary to take into account both its variable and binary nature. This paper reports the results of an infrared study of the components of the T Tau system at high spatial resolution. The work includes two-dimensional nearinfrared (1-5 $\mu \mathrm{m})$ speckle imaging, mid-infrared (10-20 $\mu \mathrm{m})$ slit scans, and 1-20 $\mu \mathrm{m}$ photometry. Nearly simultaneous measurements over a large range of wavelengths together with measurements at a smaller number of wavelengths over a much longer time period provide the opportunity to study both the nature of the two components as they appeared at the end of 1990 and their behavior from 1981 to the present. The 1990 spectral energy distribution of $\mathrm{T}$ Tau $\mathrm{N}$ is fit by two components-a photosphere and a circumstellar disk. T Tau S's spectral energy distribution is dominated by luminosity from an accretion disk. The repeated measurements show a dramatic change in the brightness of $\mathrm{T}$ Tau S over the last two years, which we propose resulted from an increase in the accretion rate.

The observations are presented in Sec. 2 and the results in Sec. 3. In Sec. 4 we examine the 1-20 $\mu \mathrm{m}$ spectral energy distributions of T Tau N and T Tau S separately, as well as the variation in the flux density of T Tau S between 1981 October and 1991 January. Our conclusions are summarized in Sec. 5.

\section{OBSERVATIONS AND REDUCTION}

\subsection{Speckle Observations}

Two-dimensional near-infrared speckle observations of $\mathrm{T}$ Tau $\left[\alpha(1950)=04^{\mathrm{h}} 19^{\mathrm{m}} 04.21^{\mathrm{s}}, \delta(1950)=+19^{\circ} 25^{\prime} 05^{\prime \prime} 4\right]$ were made between 1989 August and 1990 November at the $f / 415$ Gregorian focus of the Hale $5 \mathrm{~m}$ telescope using the Cassegrain infrared camera with a Hughes-Santa Barbara Research Center $58 \times 62 \mathrm{InSb}$ hybrid array. We determined the scale and orientation of the detector by observing and reconstructing images of several binaries with well known orbits (McAlister \& Hartkopf 1988). Referred to the sky, the pixel scale is $0.053 \times 0.053( \pm 0.001)$ and the field of view of the array is 3 ". $1(\mathrm{E}-\mathrm{W}) \times 3$ ". $3(\mathrm{~N}-\mathrm{S})$. Thus the data were slightly undersampled for all wavelengths less than 2.6 $\mu \mathrm{m}$.

Speckle measurements were made in the photometric $H(1.6 \mu \mathrm{m}), K(2.2 \mu \mathrm{m})$, and $L^{\prime}(3.7 \mu \mathrm{m})$ bands, and in additional narrow bands $(\delta \lambda / \lambda=0.015)$ at 3.3 and $4.8 \mu \mathrm{m}$. Table 1 contains the details of the speckle observations. The observations were carried out in sets of object/calibrator "pairs." The term "pair" refers to 400 consecutive short exposure frames of the T Tau system followed by 400 exposures of a nearby calibrator star, which is assumed to be unresolved. Estimates of the errors introduced by changing atmospheric conditions between any two observations were obtained by examining multiple object/calibrator pairs. The use of two calibration stars for some of the observations ensured that at least one would be unresolved. In fact, all the calibrators for these observations were unresolved at the diffraction limit of the $5 \mathrm{~m}$ telescope. At each wavelength the FWHM of integrated calibrator data provided a measure of the seeing and is reported in Table 1.

Diffraction-limited Fourier amplitudes were recovered by deconvolving the ensemble-averaged autocorrelation (the Fourier equivalent of the power spectrum) of T Tau with that of an unresolved calibrator (cf. Labeyrie 1970; Gorham 
TABLE 1. Journal of speckle observations of T Tau.

\begin{tabular}{cccccccc}
\hline \hline Date & $\lambda_{o}$ & $\delta \lambda$ & Cal\#1 & Cal\#2 & Exp. Time & Pairs & Seeing $(\lambda)^{a}$ \\
UT & $\mu m$ & $\mu m$ & SAO & SAO & sec & & $n$ \\
\hline 1989 Aug 16 & 2.2 & 0.4 & 76548 & $\ldots$ & 0.10 & 21 & 0.9 \\
1989 Aug 17 & 1.65 & 0.3 & 76548 & $\ldots$ & 0.10 & 16 & 0.7 \\
1989 Dec 9 & 2.2 & 0.4 & 93918 & $\ldots$ & 0.10 & 7 & 1.0 \\
1990 Aug 7 & 2.2 & 0.4 & 93918 & $\ldots$ & 0.10 & 2 & 0.7 \\
1990 Oct 3 & 3.7 & 0.64 & 93918 & 93974 & 0.05 & 4 & 0.8 \\
1990 Oct 3 & 4.8 & 0.07 & 93897 & $\ldots$ & 0.10 & 7 & 0.8 \\
1990 Oct 3 & 3.28 & 0.05 & 93897 & $\ldots$ & 0.10 & 4 & 0.9 \\
1990 Nov 8 & 4.8 & 0.07 & 93897 & $\ldots$ & 0.20 & 5 & 1.1 \\
1990 Nov 9 & 2.2 & 0.4 & 93918 & 93874 & 0.10 & 7 & 1.3 \\
1990 Nov 9 & 1.65 & 0.3 & 93918 & 93874 & 0.07 & 8 & 1.2 \\
\hline \hline
\end{tabular}

Note to TABLE 1

(a) The seeing is reported as the FWHM of the calibrator at the observed wavelength.

et al. 1990). The Fourier phases of T Tau were retrieved by first calculating the bispectrum of the object (Lohmann et al. 1983) and then fitting the bispectrum phases using a global least-squares algorithm (Cornwell 1987; Gorham et al. 1989). A Fourier inversion of these amplitudes and phases produced a reconstructed image of T Tau for every object/ calibrator pair obtained. The final images presented in Sec. 3 are averages of all the pairs reconstructed for each observation. Previous papers describe the application of this technique in more detail (Gorham et al. 1989; Ghez et al. 1990).

\subsection{Slit Scans}

Simultaneous north-south near-infrared and mid-infrared slit scans of T Tau were obtained at the $f / 70$ Cassegrain focus of the Hale $5 \mathrm{~m}$ telescope in 1985 December, 1990 October, and 1991 January (see Table 2) to spatially separate the two components of T Tau at mid-infrared wave-

TABLE 2. Log of slit scan observations of T Tau

\begin{tabular}{ccccc}
\hline \hline Date & $\lambda$ & $\delta \lambda$ & NIR Ref $\lambda$ & Conditions \\
& $\mu m$ & $\mu m$ & $\mu m$ & \\
\hline 1985 Dec 21 & 10.1 & 5.0 & 2.2 & photometric \\
1990 Oct 6 & 10.1 & 5.0 & 1.65 & thin cirrus \\
1991 Jan 6 & 10.1 & 5.0 & 1.65 & cirrus \\
1991 Jan 6 & 20 & 6.0 & 1.65 & cirrus \\
1991 Jan 6 & 8.7 & 0.1 & 1.65 & cirrus \\
1991 Jan 6 & 9.5 & 0.1 & 1.65 & cirrus \\
1991 Jan 6 & 11.2 & 0.2 & 1.65 & cirrus \\
1991 Jan 6 & 12.5 & 0.2 & 1.65 & cirrus \\
\hline \hline
\end{tabular}

lengths. The near-infrared scans delineated the optical component, $\mathrm{T}$ Tau $\mathrm{N}$ and therefore provided a reference to coadd the noisier mid-infrared scans (Matthews et al. 1987), taken with broadband 10 and $20 \mu \mathrm{m}$ filters, as well as narrowband filters in the silicate feature $(\lambda=8.7,9.5,11.2$, and 12.5 $\mu \mathrm{m})$. Figure 1 shows the 1985 and 1990 coadded slit scans of $\mathrm{T}$ Tau at $10 \mu \mathrm{m}$.

At all wavelengths the slit scans of the point source $\alpha$ Tau, which were interspersed with those of T Tau, determined the point-spread function of the telescope. One-dimensional images of the $T$ Tau binary together with the flux density ratio of the two components were obtained at each wavelength by fitting two of the observed point spread functions appropriately separated in the north-south direction to the coadded T Tau slit scans.

\subsection{Photometric Observations}

Photometric measurements of $\mathrm{T}$ Tau were made in the photometric bands $J(1.25 \mu \mathrm{m}), H(1.65 \mu \mathrm{m}), K(2.2 \mu \mathrm{m})$, $L^{\prime}(3.7 \mu \mathrm{m}), M(4.8 \mu \mathrm{m}), N(10.1 \mu \mathrm{m})$, and $Q(20 \mu \mathrm{m})$ on 1990 October 5 and 1990 December 31 with a single element InSb detector and a Ge:Ga bolometer at the $f / 70$ Cassegrain focus of the Hale $5 \mathrm{~m}$ telescope. Beam diameters were $5^{\prime \prime}$ for the $J, H, K, L^{\prime}$, and $M$ observations and 4.6 for the $N$ and $Q$ observations. Sky subtraction was carried out by chopping $15^{\prime \prime}$ north and south of the object. The stars listed in Elias $e t$ al. (1982) and $\alpha$ Tau were used for photometric calibration. Additional photometry of T Tau was obtained in the nearinfrared bands on 1991 February 24 using the InSb array at
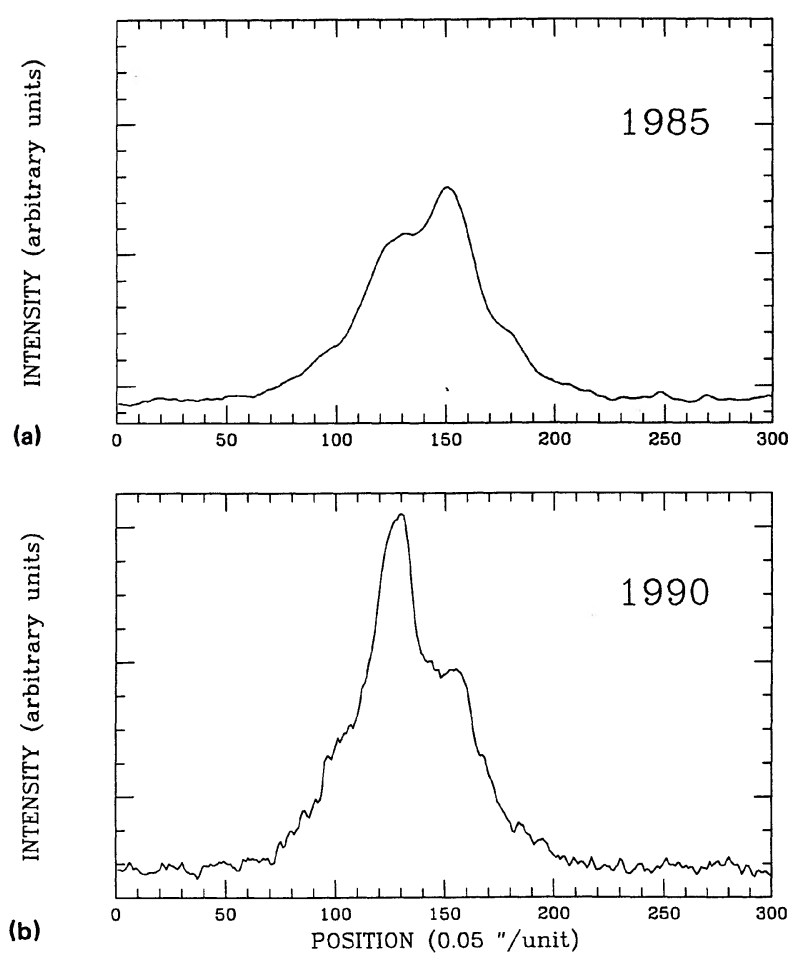

FIG. 1. Coadded slit scans of T Tau at $10 \mu \mathrm{m}$. North is to the right in both figures. (a) Data obtained in 1985, show T Tau N dominating the system. (b) In 1990, T Tau S dominates the system. These slit scans were fit with two point spread functions, determined by slit scans of a point source, to obtain flux ratios. 
the $f / 70$ Cassegrain focus of the Hale $5 \mathrm{~m}$ telescope and at 10 and $2 \mu \mathrm{m}$ from slit scans taken under photometric conditions on 1985 December 21. Table 3 lists the photometric results.

\section{RESULTS}

\subsection{Speckle Image Reconstructions}

The speckle images of the T Tau system at wavelengths between 1 and $5 \mu \mathrm{m}$ are presented in Figs. 2(a)-2(e) and show that the system is a binary with separation $0.73 \pm 0.01$ and position angle $176^{\circ} \pm 1^{\circ}$ measuring the southern component, $\mathrm{T} \mathrm{Tau} \mathrm{S}$, with respect to the northern component, $\mathrm{T}$ Tau N. In the images reconstructed from data obtained between October and November 1990, the northern component dominates the system at all wavelengths shorter than 3 $\mu \mathrm{m}$. Given that the southern source becomes fainter at shorter wavelengths, it is sensible to assume that it continues to decrease in brightness at visual wavelengths, and we therefore identify the northern source with the optical star as expected from earlier astrometric results (Hanson et al. 1983).

None of the near-infrared images reveal the third component, T Tau O' (Nisenson et al. 1985; Maihara \& Kataza 1991). The limits for its detection in these images were set by calculating the rms noise in the region where $\mathrm{T} T \mathrm{Tau} \mathrm{O}^{\prime}$ first appeared; a further discussion of these limits is in Gorham $e t$ al. (1991).

\subsection{Flux Density Ratios}

The near-infrared flux density ratios between $\mathrm{T}$ Tau $\mathrm{N}$ and $\mathrm{T}$ Tau $\mathrm{S}$ and the errors were determined by fitting a fringe pattern to the recovered power spectra. A binary with flux ratio $R$ and separation $s$ produces a fringe pattern $P(\mathbf{x})=P^{2}+1+2 R \cos (2 \pi \mathbf{s} \cdot \mathbf{x})$ in the power spectrum (see Fig. 3). At mid-infrared wavelengths the flux density ratios and errors were obtained from fits to the slit scans as discussed in Sec. 2. Table 4 lists the flux density ratios at near- and mid-infrared wavelengths.

\subsection{Flux Densities of T Tau N and T Tau $S$}

The combination of photometric data for the total system (Table 3 ) and the measured flux ratios determined the absolute brightness of the separate components, $\mathrm{T}$ Tau $\mathrm{N}$ and $\mathrm{T}$ Tau S, given in Table 4. If photometric data were not obtained contemporaneously with the measurements of the flux ratios, photometric observations obtained within one

TABLE 3. Photometry of the total T Tau system.

\begin{tabular}{cccccc}
\hline \hline & $1985 \operatorname{Dec} 21$ & 1990 Oct 5 & $1990 \mathrm{Dec} 31$ & $1991 \mathrm{Feb} 24$ & $1991 \mathrm{Mar} 30$ \\
\hline$\lambda(\mu m)$ & $F_{\mathrm{v}}(J y)$ & $F_{\mathrm{v}}(J y)$ & $F_{\mathrm{v}}(J y)$ & $F_{\mathrm{v}}(J y)$ & $F_{\mathrm{v}}(J y)$ \\
$\mathrm{J}(1.3)$ & $\ldots$ & $2.3 \pm 0.1$ & $2.2 \pm 0.1$ & $2.2 \pm 0.1$ & $\ldots$ \\
$\mathrm{H}(1.6)$ & $\ldots$ & $3.4 \pm 0.2$ & $3.3 \pm 0.2$ & $3.6 \pm 0.2$ & $\ldots$ \\
$\mathrm{K}(2.2)$ & $3.5 \pm 0.4$ & $5.6 \pm 0.3$ & $5.4 \pm 0.3$ & $5.5 \pm 0.3$ & $\ldots$ \\
$\mathrm{L}(3.7)$ & $\ldots$ & $12.2 \pm 0.8$ & $11.7 \pm 0.7$ & $11.9 \pm 0.8$ & $\ldots$ \\
$\mathrm{M}(4.8)$ & $\ldots$ & $18 \pm 1$ & $14.8 \pm 0.9$ & $\ldots$ & $\ldots$ \\
$\mathrm{N}(10.1)$ & $11.6 \pm 2$ & $28 \pm 3$ & $\ldots$ & $\ldots$ & $23 \pm 2$ \\
$\mathrm{Q}(20)$ & $\ldots$ & $45 \pm 7$ & $\ldots$ & $\ldots$ & $38 \pm 6$ \\
\hline \hline
\end{tabular}
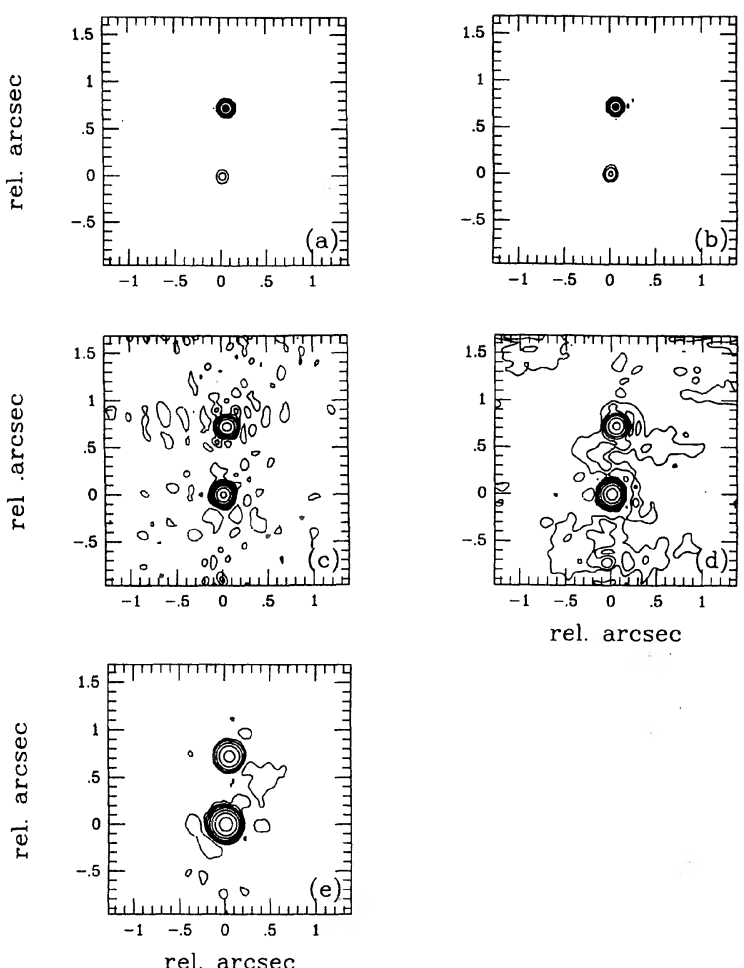

FIG. 2. Speckle image reconstructions of $T$ Tau. The images shown have been centered on a point midway between the two components. North is up and east is to the left and the contour levels are $-1 \%, 1 \%, 2 \%, 4 \%$, $8 \%, 16 \%, 32 \%, 64 \%$ of the peak value in each map. (a) The $1.6 \mu \mathrm{m}$ image reconstructed from data obtained 1990 November 9. Although the secondary looks slightly resolved, this is most likely an artifact of undersampling. (b) The $2.2 \mu \mathrm{m}$ image reconstructed from data obtained 1989 August 16. (c) The $3.28 \mu \mathrm{m}$ image reconstructed from data obtained 1990 October 3. (d) The $3.7 \mu \mathrm{m}$ image reconstructed from data obtained 1990 October 3 . The increased noise at the position angle $180^{\circ}$ from the secondary is the result of slightly different amplitude and phase solutions for the binary's flux ratio. (e) The $4.8 \mu \mathrm{m}$ image reconstructed from data obtained 1990 October 3 . The low frequency noise is characteristic of a seeing mismatch between the $T$ Tau observations and those of its calibrator.

month were used. The near-infrared flux of the T Tau system prior to 1990 October was estimated by integrating the speckle data and calculating the relative strength of $T$ Tau to that of the calibrator. The calibrator stars are of spectral type F0 and B8, and typically remain constant over timescales of many years. Thus photometric observations of the speckle calibrators obtained in 1991 February provided a measure of the earlier 1.6 and $2.2 \mu \mathrm{m} \mathrm{T} \mathrm{Tau} \mathrm{total} \mathrm{flux} \mathrm{densities.} \mathrm{An}$ interpolation between the photometric values in $K$ and $L^{\prime}$ bands gave an estimate of the flux density of the system at 3.3 $\mu \mathrm{m}$.

\subsection{Silicate Measurements for $T$ Tau $N$ and $T$ Tau $S$}

The 1991 January 6 slit scans of T Tau at silicate wavelengths using narrowband filters yielded reliable flux density ratios, but due to the presence of cirrus, the photometric measurements were poor. Subsequent photometric measurements of $\mathrm{T}$ Tau at mid-infrared wavelengths, using both broadband and narrowband filters, were made on 1991 

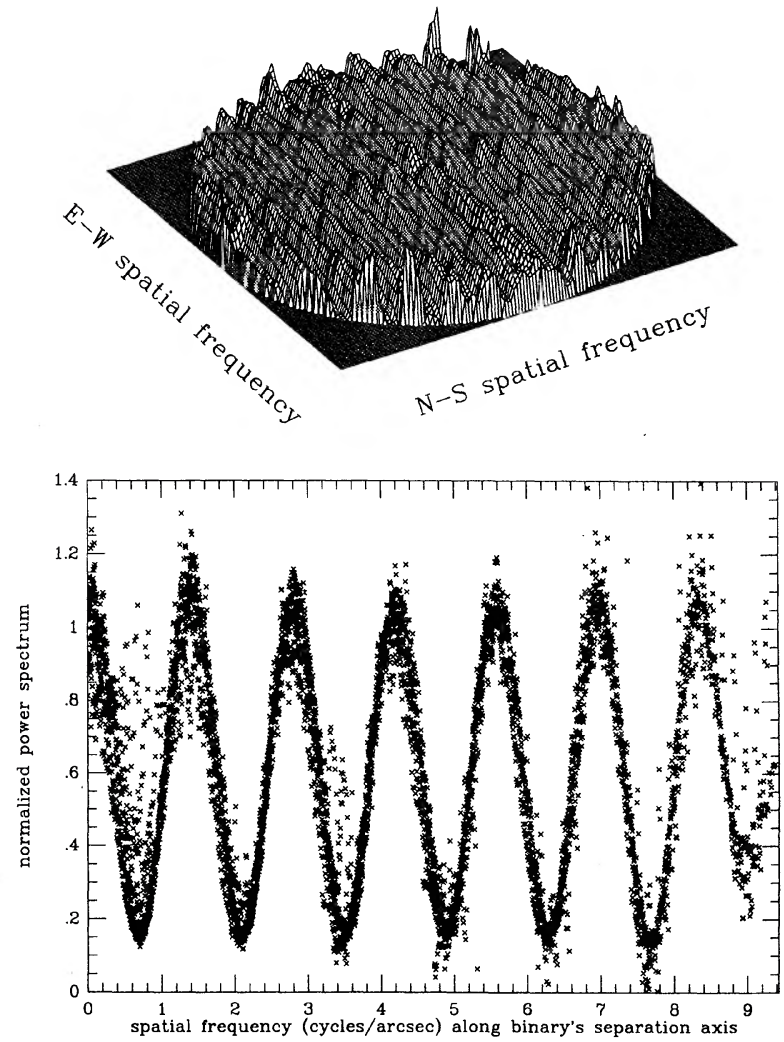

Fig. 3. T Tau's spatial power spectrum. (a) The two-dimensional power spectrum obtained from data obtained at $2.2 \mu \mathrm{m}$ on 9 November 1990. (b) The two-dimension visibility function is collapsed along the axis of the binary's separation. A binary separated by $\mathrm{s}$ with a flux ratio of $R$ will produce a power spectrum of the form $P(\mathbf{x})=R^{2}+1+2 R \cos (2 \pi \mathbf{s} \cdot \mathbf{x})$. The binary is clearly observed in this power spectrum out to the highest spatial frequencies obtained by the $5 \mathrm{~m}$ telescope. The increased scatter at low spatial frequencies is due to seeing miscalibration (i.e., slightly different seeing conditions between the time $\mathrm{T}$ Tau and its calibrator were observed).

March 30 . The 10 and $20 \mu \mathrm{m}$ broadband photometric measurements showed a $20 \%$ decrease from the previous measurements obtained on 1990 October 5. We assumed that this change resulted entirely from a "colorless" change in T Tau $\mathrm{S}$ (see the discussion and Fig. 6) and derived the absolute flux densities of the T Tau system on 1991 January 6 at the silicate feature wavelengths from the 1991 March 30 measurements. Table 4 lists the values of the silicate flux densities of $\mathrm{T}$ Tau $\mathrm{N}$ and $\mathrm{T}$ Tau $\mathrm{S}$ obtained by combining the estimated photometry and the measured flux ratios.

\section{DISCUSSION}

At the distance to the Taurus dark cloud complex, $140 \mathrm{pc}$ (Elias 1978), the projected angular separation, $0.73 \pm 0$ ".01, between the components of $\mathrm{T}$ Tau corresponds to a linear distance of $102 \mathrm{AU}$. The current measurement of the separation in conjunction with previously reported measurements listed in Table 5 cannot distinguish between the two stars being gravitationally bound or simply members of the same cloud (Jones \& Herbig 1979). If T Tau S is a distant background object, then it would appear stationary and the relative separation of the two stars over time should decrease (see Table 5), reflecting the proper motion of $\mathrm{T}$ Tau $\mathrm{N}$,

\begin{tabular}{cccccc}
\multicolumn{5}{c}{ TABLE 4. Photometry of T Tau components. } \\
\hline \hline$\lambda$ & Date & Observation & Flux Density Ratio & $f_{\mathrm{v}}($ TTau $N)$ & $f_{\mathfrak{v}}($ TTau $S)$ \\
$\mu m$ & UT & & $f_{\mathrm{v}}($ TTau $N) / f_{\mathrm{v}}($ TTau $S)$ & $\mathrm{Jy}$ & $\mathrm{Jy}$ \\
\hline 1.6 & 1989 Aug 17 & Speckle & $80 \pm 30$ & $3.2 \pm 1.3$ & $0.04 \pm 0.02$ \\
1.6 & 1990 Nov 9 & Speckle & $10.0 \pm 0.4$ & $3.1 \pm 0.2$ & $0.31 \pm 0.02$ \\
2.2 & 1989 Aug 16 & Speckle & $6.2 \pm 0.2$ & $3.9 \pm 0.5$ & $0.6 \pm 0.07$ \\
2.2 & 1989 Dec 9 & Speckle & $2.71 \pm 0.05$ & $3.9 \pm 0.4$ & $1.4 \pm 0.1$ \\
2.2 & 1990 Aug 7 & Speckle & $2.20 \pm 0.06$ & $3.8 \pm 0.5$ & $1.7 \pm 0.2$ \\
2.2 & 1990 Nov 9 & Speckle & $2.40 \pm 0.05$ & $3.9 \pm 0.2$ & $1.60 \pm 0.07$ \\
3.3 & 1990 Oct 3 & Speckle & $0.43 \pm 0.04$ & $3.1 \pm 0.4$ & $7.2 \pm 0.9$ \\
3.7 & 1990 Oct 3 & Speckle & $0.44 \pm 0.01$ & $3.7 \pm 0.3$ & $8.5 \pm 0.6$ \\
4.8 & 1990 Oct 3 & Speckle & $0.244 \pm 0.006$ & $3.6 \pm 0.3$ & $14.7 \pm 1.1$ \\
4.8 & 1990 Nov 8 & Speckle & $0.24 \pm 0.02$ & $3.2 \pm 0.3$ & $13.3 \pm 1.3$ \\
8.7 & 1991 Jan 6 & Slit scan & $0.21 \pm 0.02$ & $4.5 \pm 0.8$ & $22 \pm 4$ \\
9.5 & 1991 Jan 6 & Slit scan & $0.60 \pm 0.03$ & $8.7 \pm 1.5$ & $14 \pm 2$ \\
11.2 & 1991 Jan 6 & Slit scan & $0.33 \pm 0.07$ & $7 \pm 1$ & $22 \pm 4$ \\
12.5 & 1991 Jan 6 & Slit scan & $0.27 \pm 0.03$ & $7 \pm 1$ & $27 \pm 4$ \\
10.1 & 1985 Dec 21 & Slit scan & $2.6 \pm 0.06$ & $8.4 \pm 1.1$ & $3.2 \pm 0.4$ \\
10.1 & 1990 Oct 6 & Slit scan & $0.50 \pm 0.03$ & $8.5 \pm 1.1$ & $17.1 \pm 2.3$ \\
10.1 & 1991 Jan 6 & Slit scan & $0.41 \pm 0.01$ & $8.3 \pm 1.0$ & $20.4 \pm 2.5$ \\
20 & 1991 Jan 6 & Slit scan & $0.46 \pm 0.03$ & $14.3 \pm 2.3$ & $31 \pm 5$ \\
\hline \hline & & & & & \\
\hline
\end{tabular}

$\mu_{\alpha}=0.009 \pm 0.001 \mathrm{yr}^{-1}$ and $\mu_{\delta}=0.014 \pm 0.001 \mathrm{yr}^{-1}$ (Jones \& Herbig 1979), as opposed to the observed increase. Thus we conclude that $\mathrm{T}$ Tau $\mathrm{S}$ is not a distant background object. We will continue to assume, as others have done, that the two components form a physical pair on the basis of statistics (Dyck et al. 1982) and the $2 \mathrm{~cm}$ bridge of emission connecting the two sources (Schwartz et al. 1986).

The spectral energy distributions of the components of $T$ Tau shown in Fig. 4 were constructed by combining the photometric, speckle, and slit scan results obtained during the period 1990 October to 1991 January. Additional visual

TABLE 5. Relative separations.

\begin{tabular}{ccc||cc|cc}
\hline \hline \multicolumn{3}{c||}{ Measurements } & \multicolumn{4}{c}{ Predictions for 1990.9 } \\
\hline Sepn & PA & Epoch & Sepn & $P A^{a}$ & $S e p n^{b}$ & $P A^{b}$ \\
$\operatorname{arcsec}$ & deg & $1900+$ & $\operatorname{arcsec}$ & $\operatorname{deg}$ & $\operatorname{arcsec}$ & deg \\
$0.6 \pm 0.1$ & $\mathrm{~N}-\mathrm{S}$ & $81^{c}$ & $0.5 \pm 0.1$ & $\mathrm{~N}-\mathrm{S}$ & $0.7 \pm 0.1$ & $\mathrm{~N}-\mathrm{S}$ \\
$0.55 \pm 0.07$ & $167 \pm 2$ & $82.5^{d}$ & $0.42 \pm 0.07$ & $174 \pm 2$ & $0.6 \pm 0.1$ & $171 \pm 6$ \\
$0.65 \pm 0.07$ & $174.7 \pm 0.7$ & $83.75^{c}$ & $0.55 \pm 0.07$ & $180.4 \pm 0.8$ & $0.66 \pm 0.09$ & $177 \pm 4$ \\
$0.73 \pm 0.03$ & $176 \pm 1$ & $90.9^{\prime}$ & $\ldots$ & $\ldots$ & $\ldots$ & $\ldots$ \\
\hline \hline
\end{tabular}

Notes to TABLE 5

(a) The predicted relative position of the two components if T Tau S remained stationary and $\mathrm{T}$ Tau $\mathrm{N}$ moved according to its proper motion. (b) The predicted separation if $\mathrm{T}$ Tau $\mathrm{N}$ moved according to its proper motion and $\mathrm{T}$ Tau $\mathrm{S}$ had proper motion consistent with being a member of the Taurus Dark Cloud (Jones \& Herbig 1979).

(c) Near-infrared measurement by Dyck et al. (1982).

(d) Radio measurement by Schwartz et al. (1984).

(e) Radio measurement by Schwartz et al. (1986)

(f) This work. 


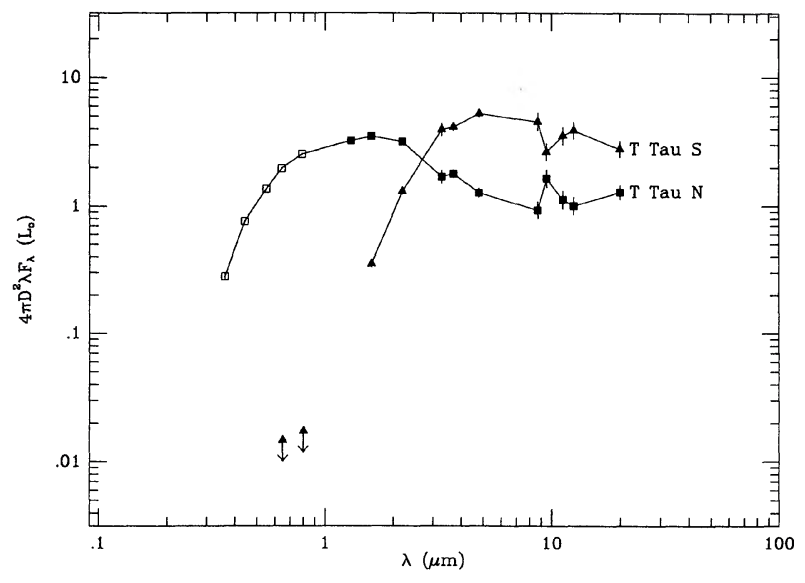

FIG. 4. The spectral energy distribution of $T$ Tau $N$ (square points) and $T$ Tau $S$ (triangular points). The filled points are data presented in this paper and the unfilled points are optical photometry measurements provided by Herbst. Optical speckle measurements (Gorham et al. 1991) provided limits on the detection of $\mathrm{T}$ Tau S, and justified the assignment of the optical photometry measurements to $T$ 'Tau $N$. In the wavelength range $0.8-20 \mu \mathrm{m}$, $\mathrm{T}$ Tau $\mathbf{S}$ contributes twice as much luminosity as $\mathrm{T}$ Tau $\mathbf{N}$ to the total luminosity of the T Tau system. Note that the $10 \mu \mathrm{m}$ silicate feature appears to be in emission for $\mathrm{T}$ Tau $\mathrm{N}$ and in absorption for $\mathrm{T}$ Tau $\mathrm{S}$. The lines are drawn only to guide the eye.

photometry from early 1990, provided by Herbst (1991) and assigned to $\mathrm{T} T$ Tau N, and limits for T Tau S at 0.6 and 0.8 $\mu \mathrm{m}$ from speckle interferometry in 1990 September (Gorham et al. 1991) are also included in this plot. Both the inferred photometry obtained from the 1990 optical speckle data and the AAVSO database of visual observations suggest that $\mathrm{T}$ Tau $\mathrm{N}$ has remained at roughly constant visual flux densities over this period.

The spectral energy distribution of $\mathrm{T}$ Tau S shown in Fig. 4 indicates that it contributes a significant fraction of the overall infrared excess. T Tau N, however, still has its own infrared excess. The measured luminosity of $\mathrm{T} T \mathrm{Tau} S$ from 0.8 to $20 \mu \mathrm{m}$ is $15 L_{0}$, a factor of 2 greater than that of T Tau $\mathrm{N}, 7.5 L_{0}$. An estimate of the bolometric luminosity of the individual components is difficult since measurements made at other longer wavelengths (e.g., the IRAS observations) did not spatially resolve the system and, additionally, $\mathrm{T}$ Tau S's flux density has changed in the recent past (see Figs. 1 and 7).

\subsection{T Tau N and its Circumstellar Material}

Based on the speckle limits for the detection of $\mathrm{T} T \mathrm{Tau} S$ at visual wavelengths, it is reasonable to assume that an optical spectrum of the T Tau system is due entirely to T Tau N. Previous measurements of the T Tau system's optical spectrum indicated the presence of a K0 star (Cohen \& Kuhi 1979; Bertout et al. 1988). We assume an effective temperature of a K0 star (Johnson 1965) and fit the visual data with a $8.8 L_{0}$ blackbody reddened by $A_{v}=1.5 \mathrm{mag}$ and the averaged extinction law given by Savage \& Mathis (1979) (Fig. 5 ). These parameters agree quite well with what have been previously used by others (Adams et al. 1988; Cohen et al. 1989).

Adams et al. (1987) and others propose that the presence of a circumstellar disk accounts for the infrared excess ob-

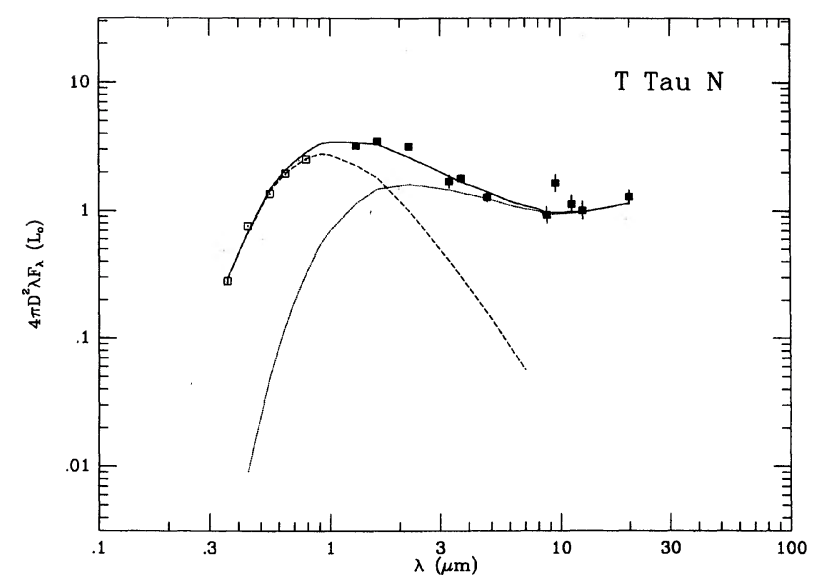

FIG. 5. The spectral energy distribution of T Tau N. The dashed line is a $8.8 L_{0}$ blackbody with an effective temperature of a $\mathrm{K} 0$ star reddened by $A_{v}=1.5$ mag representing the photosphere of the star. A power law temperature distribution plus stellar heating disk model (dotted line) accounts for the infrared excess. The solid curve is the sum of the photospheric blackbody and the disk model.

served in most $\mathrm{T}$ Tauri stars. T Tau N's mid-infrared data excess suggests the use of a disk model with a power law temperature distribution, $T(r)=T_{0}(r / 1 \mathrm{AU})^{-q}$ plus heating from the central star (Beckwith et al. 1990). This model with $T_{0}=260, q=0.42$, and an inner disk radius of $4.3 R_{0}$ accounts for the observed infrared excess. Figure 5 shows the blackbody representation of the photosphere, the disk model, and the sum of the two which accommodates the general shape of the spectral energy distribution quite well. The heating of the inner portions of the disk by the central star provides the extra luminosity seen at near-infrared wavelengths.

The $10 \mu \mathrm{m}$ silicate feature appears to be in emission in $\mathrm{T}$ Tau N's spectral energy distribution. This implies that the radiation at $10 \mu \mathrm{m}$ is not optically thick. Since material at temperatures of roughly $330 \mathrm{~K}$ contribute most to the $10 \mu \mathrm{m}$ radiation, a corresponding location of roughly $120 R_{0}$ in the disk is found from the model temperature distribution. Thus, at this distance from the central star, the circumstellar material is no longer optically thick.

\subsection{T Tau S and its Circumstellar Material}

$\mathrm{T}$ Tau $\mathrm{S}$ does not appear to be a clump of material heated solely by T Tau N. Over the wavelength range of $0.8-20 \mu \mathrm{m}$, the flux density measurements show $\mathrm{T}$ Tau $\mathrm{S}$ to be twice as luminous as T Tau N. Thus T Tau S must be a self-luminous source.

The spectral energy distribution of T Tau S shows a $10 \mu \mathrm{m}$ silicate absorption feature. The depth of the feature, $\tau_{9.5 \mu \mathrm{m}}=0.4 \pm 0.2$, corresponds to $4.6 \pm 2.5 \mathrm{mag}$ of visual extinction along the line of sight (Reike \& Lebofsky 1985). Since the line of sight to $\mathrm{T}$ Tau $\mathrm{N}$ is not obstructed by much dust, it is most likely that the dust is located in a relatively small zone around $\mathrm{T}$ Tau $\mathrm{S}$ alone.

If the visual extinction to T Tau S is $A_{v}=4.6 \pm 2.5 \mathrm{mag}$, then the near-infrared part of the spectral energy distribution can be fit by a reddened blackbody of temperature $930 \pm 50 \mathrm{~K}$, radius $102 \pm 6 R_{0}$, and luminosity $7.0 \pm 0.7 L_{0}$ this fit is shown in Fig. 6. The low effective temperature 


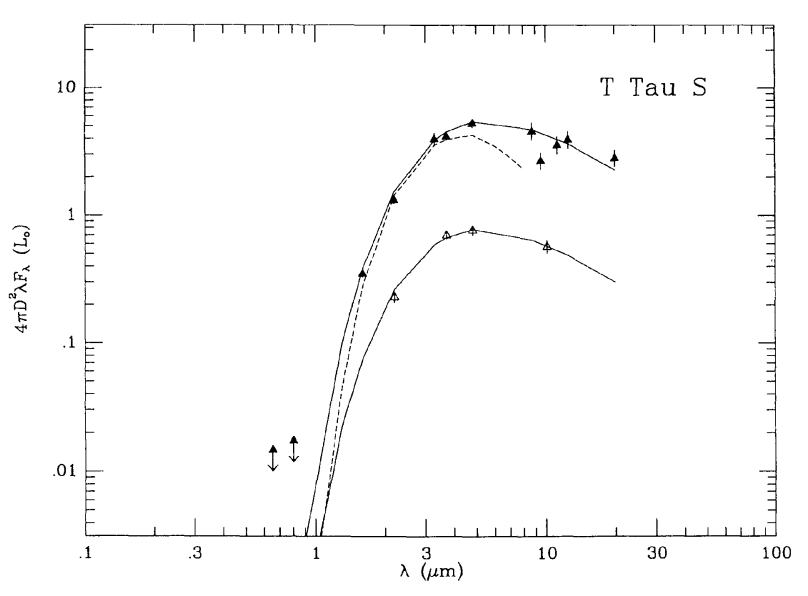

FIG. 6. The spectral energy distribution of T Tau S. The near-infrared points are fit by a reddened blackbody of temperature $930 \mathrm{~K}$ and luminosity $6.9 L_{0}$ (dashed line). The visual extinction applied, $A_{v}=4.6 \mathrm{mag}$, was obtained from the depth of the silicate feature. The solid curves show accretion disk models for both the preflare data (open triangles) and the end of 1990 data (filled triangles).

supports the protostellar model previously suggested by Hansen et al. (1983) and Bertout (1983). The spectral energy distribution of a typical protostar peaks at roughly 100 $\mu \mathrm{m}$ (Adams et al. 1987), whereas that of T Tau S peaks at 3 $\mu \mathrm{m}$. This implies that if T Tau S is a protostar it is atypical, in that it is not dominated by radiation from a mass of cold dust. This apparent lack of cold dust may stem from the binary nature of the $\mathrm{T}$ Tau system, since the outer part a protostellar dust shell might be disrupted by $\mathrm{T}$ Tau $\mathrm{N}$.

The measured flux densities of $\mathrm{T}$ Tau $\mathrm{S}$ changed by $2 \mathrm{mag}$ at all wavelengths between 1 and $10 \mu \mathrm{m}$ during the observations presented here. Figure 7 shows these data and previous measurements taken from the literature (Dyck et al. 1982; Beckwith et al. 1984; Maihara \& Kataza 1991). If the flare

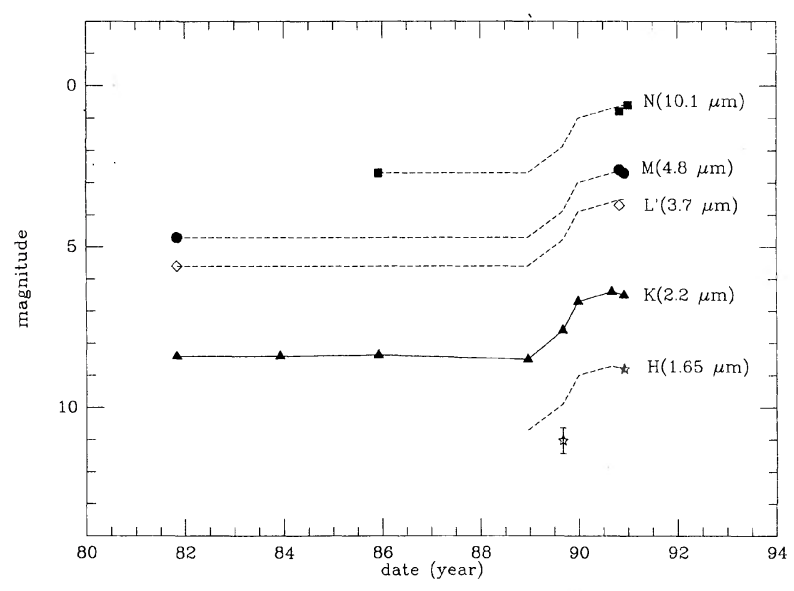

FIG. 7. The light curves for T Tau S at various wavelengths. All wavelengths show that $\mathrm{T}$ Tau $\mathrm{S}$ has undergone a 2 mag flare, and the $K$ data were obtained frequently enough to map the flare in time. The curve at $2.2 \mu \mathrm{m}$ is repeated for each wavelength to suggest that the flare was similar at all each wavelength, except $1.65 \mu \mathrm{m}$. The $K$ data shown are a compilation of the data presented here and data from the literature (Dyck et al. 1982; Beckwith et al. 1984; Maihara \& Kataza 1991). was both colorless and simultaneous at all wavelengths, then the well-sampled $K$ light curve, shifted by a constant, would fit the data at other wavelengths; see the dotted curves shown in Fig. 7. This interpretation works from 2 to $10 \mu \mathrm{m}$ but fails at $1.65 \mu \mathrm{m}$. Either the flux density of T Tau S increased more at $1.65 \mu \mathrm{m}$ than it did at other infrared wavelengths, or the flare occurred at a later time at $1.65 \mu \mathrm{m}$.

Most explanations of flares observed in young stellar sources invoke unsteady accretion from a disk surrounding the star (e.g., Bertout et al. 1988; Lin \& Papaloizou 1985). If most of the currently observed luminosity comes from accretion energy, the luminosity of the disk is $L=G M_{*} \dot{M} / 2 R_{*}$. If the star has a mass of $1 \mathscr{M}_{0}$ and a radius of $1 R_{0}$, the accretion rate derived from the 0.8 to $20 \mu \mathrm{m}$ luminosity is $3.6 \times 10^{-6} \mathscr{M}_{0} \mathrm{yr}^{-1}$. Using the $K$ light curve as a crude measure of the flare, approximately $4 \times 10^{-6} \mathscr{M}_{0}$ was accreted during the flare.

The flare suggests that $\mathrm{T}$ Tau $\mathrm{S}$ may be fit by an accretion disk model. T Tau $\mathrm{S}$ appears to be similar to FU Ori stars, which are thought to be $T$ Tauri stars experiencing rapid disk accretion and having spectral energy distributions that are dominated by an accretion disk. The accretion disk model discussed by Hartmann \& Kenyon (1985) fits the T Tau S data with an inner disk radius of $33 R_{0}$ and an accretion rate of $6 \times 10^{-6} \mathscr{M}_{0} \mathrm{yr}^{-1}$, assuming a $1 \mathscr{M}_{0}$ and $1 R_{0}$ central star. Furthermore the preflare data, the unfilled points in Fig. 6, are also well fitted by a similar model with an inner disk radius of $10 R_{0}$ and an accretion rate of $3 \times 10^{-7}$ $\mathscr{M}_{0} \mathrm{yr}^{-1}$. This implies that the flare was caused by the accretion rate increasing by a factor of 20 while the inner disk radius was pushed out by $20 R_{0}$. If T Tau $\mathrm{S}$ is an FU Ori star as opposed to a protostar, it could then possibly be coeval with T Tau N.

\section{SUMMARY}

We conclude that the T Tau system consists of two stellar components, $\mathrm{T}$ Tau $\mathrm{N}$ and $\mathrm{T}$ Tau $\mathrm{S}$, and associated disks. The optical component, T Tau N, is a $8.8 L_{0} \mathrm{~K} 0$ star, surrounded by a disk that follows a power law temperature distribution. The $10 \mu \mathrm{m}$ emission feature in T Tau N's spectral energy distribution implies that the material located at roughly $120 R_{0}$ in the disk is not optically thick.

The cool infrared companion $\mathrm{T}$ Tau $\mathrm{S}$ is assumed to have undergone a flare due to an increase in accretion. The spectral energy distribution is fit by an accretion disk model, similar to those used to explain the young outburst sources (FU Ori stars). The measured flux densities for $\mathrm{T}$ Tau $\mathrm{S}$ imply a lower limit for the accretion rate at the end of 1990 of $3.6 \times 10^{-6} \mathscr{M}_{\mathrm{o}} \mathrm{yr}^{-1}$, in agreement with the accretion disk model which predicts an accretion rate of $6 \times 10^{-6}$ $\mathscr{M}_{\mathrm{O}} \mathrm{yr}^{-1}$.

The measurements presented in this paper demonstrate the potential importance of separating close binary pairs and avoiding the confusion engendered by unresolved spectral energy distributions. In addition, the brightening of T Tau S by 2 mag at wavelengths between 1 and $10 \mu \mathrm{m}$ over a period as short as one year, illustrates the importance of obtaining simultaneous multiple wavelength observations.

We thank the staff of Palomar, especially night assistants Juan Carrasco and Will McKinley for their support during the observations, and J. Graham, A. Sargent, and T. Soifer for many helpful discussions. The Cornell observations at 
the Palomar Observatory were made as part of a continuing collaborative agreement between the California Institute of Technology and Cornell University. We thank Dr. William Herbst for kindly providing visual photometry. Tom Prince and the Caltech Conconcurrent Supercomputing Facility provided time on the Caltech NCUBE supercomputer. In- frared astrophysics at Caltech is supported by a grant from the NSF. Support for research at Cornell came from the NSF and NASA. S.R.K.'s research is supported by a Presidential Young Investigator award from the NSF, and the Packard foundation. C.A.H. is supported by a SERC/NATO research fellowship.

\section{REFERENCES}

Adams, F. C., Lada, C. J., and Shu, F. H. 1987, ApJ, 312, 788

Adams, F. C., Lada, C. J., and Shu, F. H. 1988, ApJ, 326, 865

Beckwith, S., Sargent, A., Chini, R., and Gusten, R. 1990, AJ, 99, 1024

Beckwith, S., Zuckerman, B., Skrutskie, M., and Dyck, M. 1984, ApJ, 287, 793

Bertout, C. 1983, A\&A, 126, L1

Bertout, C., Basri, G., and Bouvier, J. 1988, ApJ, 330, 350

Cohen, M., Emerson, J. P., and Beichman, C. 1989, ApJ, 339, 455

Cohen, M., and Kuhi, L. V. 1979, ApJS, 41, 743

Cornwell, T. J. 1987, A\&A, 180, 269

Dyck, H. M., Simon, T., and Zuckerman, B. 1982, ApJL, 255, L103

Elias, J. H. 1978, ApJ, 224, 857

Elias, J. H., Frogel, J. A., Matthews, K., and Neugebauer, G. 1982, AJ, 87 , 1029

Ghez, A. M., Gorham, P. W., Haniff, C. A., Kulkarni, S. R., Matthews, K., Neugebauer, G., and Weir, N. 1990, Proc. SPIE, 1237, 249

Gorham, P. W., Ghez, A. M., Kulkarni, S. R., Nakajima, T., Neugebauer, G., Oke, J. B., and Prince, T. A. 1989, AJ, 98, 1783

Gorham, P. W., Ghez, A. M., Haniff, C. A.; and Prince, T. A. 1990, AJ, 100,294

Gorham, P. W., Ghez, A. M., Haniff, C. A., Kulkarni, S. R., Matthews, K., and Neugebauer, G. 1991, AJ (submitted)

Hanson, R. B., Jones, B. F., and Lin, D. N. C. 1983, ApJL, 270, L27
Hartmann, L., and Kenyon, S. J. 1985, ApJ, 299, 462

Herbst, W. 1991, private communication

Johnson, H. L. 1965, ARA\&A, 4, 193

Jones, B. F., and Herbig, G. H. 1979, AJ, 84, 1872

Labeyrie, A. 1970, A\&A, 311, 731

Lin, D. N. C., and Papaloizou, J. C. B. 1985, Protostars and Planets II, edited by D. C. Black and M. S. Mathews (University of Arizona Press, Tucson), p. 981

Lohmann, A. W., Weigelt, G., and Wirnitzer, B. 1983, Appl. Opt., 22, 4028 Maihara, T., and Kataza, H. 1991, A\&A (in press)

Matthews, K, Neugebauer, G., McGill, J., and Soifer, B. T. 1987, AJ, 94, 297

McAlister, H. A., and Hartkopf, W. I. 1988, Second Catalog of Interferometric Measurements of Binary Stars (Center for High Angular Resolution Astronomy)

Nisenson, P., Stachnik, R. V., Karovska, M., and Noyes, R. 1985, ApJL, 297, L17

Reike, G. H., and Lebofsky, M. J. 1985, ApJ, 288, 618

Savage, B. D., and Mathis, J. S. 1979, ARA\&A, 17, 73

Schwartz, P. R., Simon, T., Zuckerman, B., and Howell, R. R. 1984, ApJL, 280, L23

Schwartz, P. R., Simon, T., and Cambell, R. 1986, ApJ, 303, 233 